\title{
HIGHER-ORDER ANALYSIS OF CRACK TIP FIELDS IN ELASTIC POWER-LAW HARDENING MATERIALS
}

\author{
L. XIA and T. C. WANG \\ LNM, Institute of Mechanics, Chinese Academy of Sciences, Beijing, 100080, P.R. China \\ and \\ C. F. SHIH \\ Division of Engineering, Brown University, Providence, RI 02912, U.S.A.
}

(Received 3 September 1992)

\begin{abstract}
A HIGHER-ORDER asymptotic analysis of a stationary crack in an elastic power-law hardening material has been carried out for plane strain, Mode I. The extent to which elasticity affects the near-tip fields is determined by the strain hardening exponent $n$. Five terms in the asymptotic series for the stresses have been derived for $n=3$. However, only three amplitudes can be independently prescribed. These are $K_{1}, K_{2}$ and $K_{5}$ corresponding to amplitudes of the first-, second- and fifth-order terms. Four terms in the asymptotic series have been obtained for $n=5,7$ and 10 ; in these cases, the independent amplitudes are $K_{1}, K_{2}$ and $K_{4}$. It is found that appropriate choices of $K_{2}$ and $K_{4}$ can reproduce near-tip fields representative of a broad range of crack tip constraints in moderate and low hardening materials. Indeed, fields characterized by distinctly different stress triaxiality levels (established by finite element analysis) have been matched by the asymptotic series. The zone of dominance of the asymptotic series extends over distances of about 10 crack openings ahead of the crack tip encompassing length scales that are microstructurally significant. Furthermore, the higher-order terms collectively describe a spatially uniform hydrostatic stress field (of adjustable magnitude) ahead of the crack. Our results lend support to a suggestion that $J$ and a measure of near-tip stress triaxiality can describe the full range of near-lip states.
\end{abstract}

\section{INTRODUCTION}

THE CHANGES in near-tip stress triaxiality as plastic flow advances beyond wellcontained yielding provide a strong argument for a two-parameter description of plane strain Mode I near-tip fields. At low loads, the near-tip stresses and deformations evolve according to a self-similar ficld. This field, characterized by a high level of stress triaxiality, also describes the development of the near-tip stresses and deformations as plastic flow progresses beyond contained yielding in so-called high-constraint crack geometries. While this high triaxiality field is only one of many possible states that can exist under fully yielded conditions, it is the only field that has received careful study until recently. When the high triaxiality field (HUTCHINSON, 1968; RICE and ROSENGREN, 1968), scaled by the $J$-integral (RICE, 1968), prevails over distances of several crack tip openings, $J$ alone sets the near-tip stress level and the size scale of the zone of high stresses and large deformations. This is the basis for correlating the 
onset of crack growth in different crack geometries with a single parameter, $J_{\mathrm{IC}}$, and for relating $J_{\mathrm{IC}}$ to fracture mechanisms operative on the microscale [see works by RICE and Johnson (1970), McMefking (1977), MCMeEking and Parks (1979), Shih and German (1981) and Needleman and Tvergaard (1983), and review articles by Hutchinson (1983), Ritchie and Thompson (1985), and Parks (1992)].

Arguments that a single parameter might not suffice to characterize the dissimilar near-tip states observed in fully yielded crack geometries have been raised by MCCLintock (1971). He noted that non-hardening plane strain crack tip fields of fully yielded bodies are not unique but exhibit levels of stress triaxiality that depend upon crack geometry. Since stress triaxiality and deformation are weakly coupled under fully yielded conditions, it may be argued that the description of near-tip states must require more than one parameter in general.

An investigation along this direction was made by $L_{I}$ and $W_{A N G}$ (1986). They performed a two-term asymptotic analysis for power-law hardening materials and proposed that $J$ and a second parameter $k_{2}$ could describe crack tip fields of varying stress triaxiality. Here $k_{2}$ is the amplitude of the second-order stress field whose value can be determined by matching the two-term expansion with full-field solutions of crack geometries. A higher-order analysis of crack tip fields has been carried out by Sharma and Aravas (1991) taking account of possible elasticity effects. However, it is apparent that more than two terms in the asymptotic series are required to match representative descriptions of complete fields near the crack tip.

Another two-parameter approach has its origin in studies by LARSSON and CARLSSON (1973) and RICE (1974) who employed a modified boundary layer (MBL) formulation to investigate the effect of the elastic $T$-stress on the elastic-plastic fields in the vicinity of the crack tip. BILBY et al. (1986) have performed large deformation analysis for a non-hardening material. They found that the fields which evolve near blunting tips in finite width geometries are similar to those which exist near the tip of a crack loaded by $J$ and T. Betegón and Hancock (1991), Al-Ani and Hancock (1991), and DU and HANCOCK (1991) investigated hardening and non-hardening materials. They correlated near-tip states in tension and bend geometries with a family of near-tip fields generated by using the MBL formulation. These investigators have proposed to parameterize crack tip stress triaxiality in fully yielded crack geometries in terms of the elastic $T$-stress (see PARKs, 1992).

The character of the high and low triaxiality stress fields surrounding the finite strain zone has been examined by O'DowD and SHIH (1991, 1992a). They undertook a full-field finite deformation analysis and systematically investigated the difference field, defined as the full-field solution less the HRR field. They found that the difference field, constructed for the full range of MBL loadings, describes a spatially uniform hydrostatic stress field (of adjustable level) ahead of the crack tip; furthermore, the difference ficld cxists over a broad range of plastic yielding in finite-width crack geometries. The implication of their finding is this: $J$, a deformation parameter, and $Q$, a stress triaxiality parameter, can characterize the full range of near-tip states surrounding the fracture process zone. SHIH et al. (1991) also observed that the difference field, which is precise in the context of MBL loadings, can be regarded as equivalent to the sum of second-and higher-order terms of an asymptotic expansion.

The above findings provided the impetus for this study. A rigorous eigen-expansion 
for an elastic power-law hardening material is performed. Up to five terms in the asymptotic series have been derived. For a moderate to low hardening material, it is shown that four terms in the asymptotic series (only three amplitudes are independent) can consistently reproduce the full range of near-tip states constructed by the MBL formulation. Moreover, the higher-order terms collectively describe a uniform hydrostatic stress field ahead of the crack tip.

\section{2. $J-Q$ THEORY}

The central points of the $J-Q$ theory are summarized here to provide the background for subsequent sections.

\subsection{Q-family of fields-MBL formulation}

The existence of the $Q$-family of fields can be made hy appealing to a modified boundary layer (MBL) formulation in which the remote tractions are given by the two-term WiLLIAMs' (1957) expansion:

$$
\sigma_{\beta \lambda}=\frac{K_{\mathrm{I}}}{\sqrt{2 \pi r}} \tilde{f}_{\beta \lambda}(\theta)+T \delta_{1 \beta} \delta_{1 \lambda .} .
$$

Here $r$ and $\theta$ are polar coordinates centered at the crack tip with $\theta=0$ corresponding to the line ahead of the crack. Within the MBL formulation and for plane strain deformation,

$$
J=\frac{1-v^{2}}{E} K_{1}^{2},
$$

where $E$ is Young's modulus, $v$ is Poisson's ratio and $J$ is RicE's (1968) $J$-integral.

Crack tip fields characterized by different triaxiality levels can be induced by loading to different values of $T$. Let $\sigma_{0}$ denote the material's tensile yield stress. From dimensional considerations, these fields can be organized into a family of crack tip ficlds parameterized by $T / \sigma_{0}$ :

$$
\sigma_{\beta \lambda}=\sigma_{0} \bar{f}_{\beta \lambda}\left(\frac{r}{J / \sigma_{0}}, \theta ; T / \sigma_{0}\right) .
$$

That is, the loading parameter $T / \sigma_{0}$ can provide a convenient means to investigate specimen geometry effects on near-tip stress triaxiality under conditions of wellcontained yielding. Indeed, such studies have been carried out by BILBY et al. (1986), BETEGÓN and HANCOCK (1991), and HARLIN and Willis (1988). Nevertheless, the result in (2.3) cannot have general applicability since the elastic solution (2.1), upon which the $T$-stress is defined, is an asymptotic condition which is increasingly violated as plastic flow progresses beyond well-contained yielding.

Recognizing the above limitation O'DowD and SHIH (1991, 1992a), henceforth referred to as OS, identified members of the family of fields by the parameter $Q$ which arises naturally in the plasticity analysis. OS write: 


$$
\sigma_{\beta i}=\sigma_{0,} f_{\beta \lambda}\left(\frac{r}{J / \sigma_{0}}, \theta ; Q\right), \quad \varepsilon_{\beta i}=\varepsilon_{0} g_{\beta \lambda}\left(\frac{r}{J / \sigma_{0}}, \theta ; Q\right), \quad u_{\beta}=\frac{J}{\sigma_{0}} h_{\beta}\left(\frac{r}{J / \sigma_{0}}, \theta ; Q\right) .
$$

The form in (2.4) constitutes a one-parameter family of self similar solutions, or in short a $Q$-family of solutions. Each member field is characterized by its level of deformation, as measured by $J / \sigma_{0}$, and by its level of crack tip stress triaxiality, as measured by $Q$, which also identifies that field as a particular member of the family. For example, the self-similar solution of RICE and JoHnson (1970), and MCMEEKING (1977) (or the HRR field), can be regarded as the $Q=0$ member field.

\subsection{Difference field and near-tip stress triaxiality}

OS considered an elastic power-law hardening material. Using the MBL formulation and by applying different values of $T / \sigma_{0}$, they generated the full range of plane strain small scale yielding solutions, designated by $\left(\sigma_{\beta \dot{\lambda}}\right)_{\mathrm{MBL}}$. OS directed attention to the difference field defined by

$$
\left(\sigma_{\beta i}\right)_{\mathrm{diff}}=\left(\sigma_{\beta \hat{\lambda}}\right)_{\mathrm{MBL}}-\left(\sigma_{\beta \lambda}\right)_{\mathrm{HRR}}
$$

where $\left(\sigma_{\beta}\right)_{\mathrm{HRR}}$ is the HRR field. They systematically investigated the character of the difference ficld within the forward sector: $|\theta|<\pi / 2, J / \sigma_{0}<r<5 J / \sigma_{01}$. This zone encompasses the microstructurally significant length scales for both brittle and ductile fracture (RITCHIE and THOMPSON, 1985).

Remarkably, the difference field in the region ahead of the crack displays minimal dependence on $r$. Furthermore, $\sigma_{r r}(\theta)_{\text {diff }} \approx \sigma_{\theta \theta}(\theta)_{\text {difl }} \approx$ constant and $\left|\sigma_{r i j}(\theta)_{\text {diff }}\right| \ll$ $\left|\sigma_{\theta \theta}(\theta)_{\text {dirr }}\right|$ in the forward sector $|\theta|<\pi / 2$ [see Figs 3, 4 and 5 in O'DowD and SHIH (1991)]. Thus an approximate representation of the difference field is

$$
\left(\sigma_{\beta \lambda}\right)_{\mathrm{ditf}}=Q \sigma_{0} \hat{\sigma}_{\beta ;}(\theta) \approx Q \sigma_{0} \delta_{\beta \lambda}, \quad|\theta|<\pi / 2
$$

where $\delta_{\beta ;}$ is the Kronecker delta. That is, the difference field ahead of the crack tip can be approximated by a spatially uniform hydrostatic stress state of adjustable magnitude. These features of the difference field are corroborated by results obtained from a higher-order asymptotic analysis which are discussed in Section 5.

To the extent that the fields derived within the MBL formulation are applicable under general yielding, a two-parameter description takes the form

$$
\sigma_{\beta i}=\left(\sigma_{\beta i}\right)_{\mathrm{HRR}}+\left(\sigma_{\beta i}\right)_{\mathrm{dirr}} \approx\left(\sigma_{\beta i}\right)_{\mathrm{HRR}}+Q \sigma_{0} \dot{\delta}_{\beta i} \text {. }
$$

The interpretation of $Q$ is transparent: negative (positive) $Q$ values imply that the hydrostatic stress ahead of the crack is reduced (increased) by $Q \sigma_{0}$ from the high triaxiality reference stress state. We emphasize that the approximate relation in $(2.7)$ is intended to facilitate the interpretation of $Q$ and its evaluation in finite width crack geometries. It does not compromise the existence of the $J-Q$ fields based on (2.4) and (2.5).

Finally, it may be noted that an admissible range of stress states for an elasticperfectly plastic material has the form 


$$
\sigma_{\beta \lambda}=\left(\sigma_{\beta i}\right)_{\text {Prandıl }}+\underbrace{Q \sigma_{0} \delta_{\beta \lambda}}_{\text {Difference field }},|\theta| \leqslant \pi / 4 .
$$

Here $\left(\sigma_{\beta i}\right)_{\text {Prindtl }}$ designates the Prandtl slip-line solution and the difference field describes a uniform hydrostatic stress scaled by $Q$ (Du and HANCOCK, 1991 ; O'DowD and SHIH, 1992a).

\section{Formulation of Asymptotic Series}

The analysis is carried out within the context of small displacement gradient theory, and the material of the cracked body is taken to be described by a Ramberg-Osgood relation and $J_{2}$ deformation theory. In uniaxial tension the material deforms according to

$$
\varepsilon / \varepsilon_{0}=\sigma / \sigma_{0}+\alpha\left(\sigma / \sigma_{0}\right)^{n}
$$

where $n$ is the strain hardening exponent, $\alpha$ a material constant, and $\sigma_{0}$ and $\varepsilon_{0}$ the reference stress and strain related through $\varepsilon_{0}=\sigma_{0} / E$ with $E$ being Young's modulus. Under multi-axial stress states, $\sigma_{i j}$, the strain is

$$
\varepsilon_{i j}=\frac{1+v}{E}-s_{i j}+\frac{1-2 v}{3 E} \sigma_{k k} \delta_{i j}+\frac{3}{2} \alpha \varepsilon_{0}\left(\frac{\sigma_{\mathrm{e}}}{\sigma_{0}}\right)^{n-1} \frac{s_{i j}}{\sigma_{0}} .
$$

Here $v$ is Poisson's ratio, $s_{i j}$ the stress deviator and $\sigma_{\mathrm{e}}=\sqrt{3 s_{i j} s_{i j} / 2}$ the effective stress.

\subsection{Plane strain equations}

We invoke the plane strain condition

$$
\varepsilon_{z}=\frac{1+v}{E}-s_{33}+\frac{1-2 v}{3 E} \sigma_{k k}+\frac{3}{2} \alpha \varepsilon_{0}\left(\frac{\sigma_{\mathrm{e}}}{\sigma_{0}}\right)^{n-1} \frac{s_{33}}{\sigma_{0}}=0,
$$

to get the relation

$$
s_{33}=-\frac{(1 / 2-v)\left(\sigma_{r}+\sigma_{0}\right)}{(3 / 2) \alpha\left(\sigma_{\mathrm{e}} / \sigma_{0}\right)^{n-1}+3 / 2},
$$

where $\sigma_{r}$ and $\sigma_{0}$ are the stress components in cylindrical coordinates. Using (3.4) in (3.2), the stress-strain relation specializes to

$$
\varepsilon_{\beta \lambda}=\frac{1+v}{E} \sigma_{\beta \lambda}+\delta_{\beta \lambda} \frac{\Gamma}{E} \sigma_{\rho p}+\frac{3}{2} \alpha\left(\frac{\sigma_{\mathrm{c}}}{\sigma_{0}}\right)^{n-1} \frac{P_{\beta \lambda}}{E} .
$$

Here, Greek indices range from 1 to $2, \sigma_{\rho \rho}=\sigma_{r}+\sigma_{\theta}, P_{\beta \lambda}=\sigma_{\beta \lambda}-\frac{1}{2} \sigma_{\rho \rho} \delta_{\beta \lambda \text {. }}$ and

$$
\Gamma=-(1+v) v+(1 / 2-v)^{2} \frac{\alpha\left(\sigma_{\mathrm{e}} / \sigma_{0}\right)^{n-1}}{\alpha\left(\sigma_{\mathrm{e}} / \sigma_{0}\right)^{n-1}+1}
$$

Since $\alpha\left(\sigma_{\mathrm{c}} / \sigma_{0}\right)^{n-1} /\left[\alpha\left(\sigma_{\mathrm{c}} / \sigma_{0}\right)^{n-1}+1\right] \rightarrow 1$ as $r \rightarrow 0$, the asymptotic value of $\Gamma$ is 


$$
\Gamma=-(1+v) v+\left(\frac{1}{2}-v\right)^{2} \text {. }
$$

The effective stress is given by

$$
\sigma_{\mathrm{e}}^{2}=\frac{3}{4}\left(\sigma_{r}-\sigma_{\theta}\right)^{2}+3 \tau_{r \theta}^{2}+\frac{9}{4} s_{33}^{2},
$$

but as $r$ asymptotically approaches 0 , the relation can be simplified to

$$
\sigma_{\mathrm{c}}^{2}={ }_{4}^{3}\left(\sigma_{r}-\sigma_{\theta}\right)^{2}+3 \tau_{r i}^{2}
$$

\subsection{Airy stress function}

Equilibrium is ensured for all stresses derived from a stress function $\phi$ :

$$
\begin{aligned}
\sigma_{r} & =\frac{1}{r}\left(\frac{\partial \phi}{\partial r}+\frac{1}{r} \frac{\partial^{2} \phi}{\partial \theta^{2}}\right), \\
\sigma_{\theta} & =\frac{\partial^{2} \phi}{\partial r^{2}}, \\
\tau_{r \theta} & =-\frac{\partial}{\partial r}\left(\frac{1}{r} \frac{\partial \phi}{\partial \theta}\right) .
\end{aligned}
$$

We attempt an asymptotic expansion of the solution in the form

$$
\phi=\sigma_{0} \sum_{i=1}^{5} K_{i} r^{s_{i}+2} \tilde{\phi}_{i}(\theta),
$$

where $s_{1}<s_{2} \ldots<s_{5} ; s_{1} \sim s_{5}$ are referred to as the stress exponents.

Substituting (3.9) into (3.8) yields

$$
\begin{aligned}
\frac{\sigma_{\beta i}}{\sigma_{0}} & =\sum_{i=1}^{5} K_{i} r^{s_{i}} \tilde{\sigma}_{\beta \lambda_{i}}(\theta) \\
& =K_{1} r^{\kappa_{1}}\left[\tilde{\sigma}_{\beta \lambda_{1}}+\xi_{1} r^{\Delta s_{2}} \tilde{\sigma}_{\beta \lambda_{2}}+\xi_{2} r^{\Delta s_{3}} \tilde{\sigma}_{\beta \lambda_{3}}+\xi_{3} r^{\Delta s_{4}} \tilde{\sigma}_{\beta \lambda_{4}}+\xi_{4} r^{\Delta s_{5}} \tilde{\sigma}_{\beta \lambda_{5}}\right],
\end{aligned}
$$

where

$$
\begin{aligned}
\tilde{\sigma}_{r_{i}} & =\ddot{\tilde{\phi}}_{i}+\left(s_{i}+2\right) \tilde{\phi}_{i}, \\
\tilde{\sigma}_{\theta_{i}} & =\left(s_{i}+2\right)\left(s_{i}+1\right) \tilde{\phi}_{i}, \quad i=1,2,3,4,5, \\
\tilde{\tau}_{r \theta_{i}} & =-\left(s_{i}+1\right) \dot{\hat{\phi}}_{i},
\end{aligned}
$$

and $\Delta s_{i}=s_{i}-s_{1}(i=2,3,4,5), \xi_{j}=K_{j+1} / K_{1}(j=1,2,3,4)$ and $\left({ }^{\circ}\right)=\mathrm{d} / \mathrm{d} \theta()$.

Now apply (3.10) to (3.7) to obtain

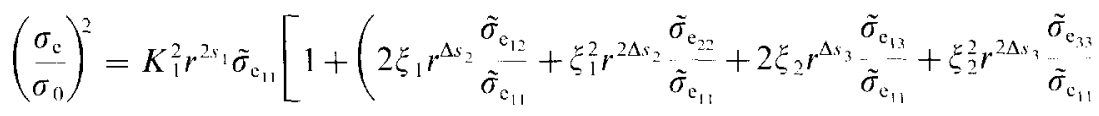

$$
\begin{aligned}
& +2 \xi_{3} r^{\Delta s_{4}} \frac{\tilde{\sigma}_{\mathrm{e}_{14}}}{\tilde{\sigma}_{\mathrm{e}_{11}}}+\xi_{3}^{2} r^{2 \Delta s_{4}} \frac{\tilde{\sigma}_{\mathrm{c}_{44}}}{\tilde{\sigma}_{\mathrm{e}_{11}}}+2 \xi_{4} r^{\Delta s_{5}} \frac{\tilde{\sigma}_{\mathrm{e}_{15}}}{\tilde{\sigma}_{\mathrm{e}_{11}}}+\xi_{4}^{2} r^{2 \Delta s_{5}} \frac{\tilde{\sigma}_{\mathrm{c}_{s 5}}}{\tilde{\sigma}_{\mathrm{e}_{11}}}
\end{aligned}
$$




$$
\begin{aligned}
& +2 \xi_{1} \xi_{2} r^{\Delta s_{2}+\Delta s_{3}} \frac{\tilde{\sigma}_{\mathrm{e}_{23}}}{\tilde{\sigma}_{\mathrm{e}_{11}}}+2 \xi_{1} \xi_{3} r^{\Delta s_{2}+\Delta s_{4}} \frac{\tilde{\sigma}_{\mathrm{e}_{24}}}{\tilde{\sigma}_{\mathrm{e}_{11}}}+2 \xi_{1} \xi_{4} r^{\Delta s_{2}+\Delta s_{5}} \frac{\tilde{\sigma}_{\mathrm{e}_{25}}}{\tilde{\sigma}_{\mathrm{c}_{11}}} \\
& \left.\left.+2 \xi_{2} \xi_{3} r^{\Delta s_{3}+\Delta s_{4}} \frac{\tilde{\sigma}_{\mathrm{e}_{34}}}{\tilde{\sigma}_{\mathrm{e}_{11}}}+2 \xi_{2} \xi_{4} r^{\Delta s_{3}+\Delta s_{5}} \frac{\tilde{\sigma}_{\mathrm{e}_{35}}}{\tilde{\sigma}_{\mathrm{e}_{11}}}+2 \xi_{3} \xi_{4} r^{\Delta s_{4}+\Delta s_{5}} \frac{\tilde{\sigma}_{\mathrm{e}_{45}}}{\tilde{\sigma}_{\mathrm{e}_{11}}}\right)\right]
\end{aligned}
$$

where

$$
\tilde{\sigma}_{\mathrm{e}_{i i}}=\frac{3}{4}\left(\tilde{\sigma}_{r_{i}}-\tilde{\sigma}_{\theta_{i}}\right)\left(\tilde{\sigma}_{r_{j}}-\tilde{\sigma}_{\theta_{j}}\right)+3 \tilde{\tau}_{r \theta_{i}} \tilde{\tau}_{r \theta_{j}}, \quad i, j=1,2,3,4,5 .
$$

In subsequent discussions the quantity $\tilde{\sigma}_{e_{11}}$ is written as $\tilde{\sigma}_{\mathbf{e}_{1}}^{2}$.

As $r \rightarrow 0$, the quantity within parentheses is much smaller than the first term in (3.12). Therefore,

$$
\begin{aligned}
\left(\frac{\sigma_{\mathrm{c}}}{\sigma_{0}}\right)^{n-1} \approx & K_{1}^{n-1} r^{(n-1) s_{1}} \tilde{\sigma}_{\mathrm{e}_{1}}^{n-1}\left\{1+\xi_{1} r^{\Delta s_{2}}(n-1) \frac{\tilde{\sigma}_{\mathrm{e}_{12}}}{\tilde{\sigma}_{\mathrm{e}_{1}}^{2}}+\xi_{2} r^{\Delta s_{3}}(n-1) \frac{\tilde{\sigma}_{\mathrm{e}_{13}}}{\tilde{\sigma}_{\mathrm{e}_{1}}^{2}}\right. \\
& +\xi_{3} r^{\Delta s_{4}}(n-1) \frac{\tilde{\sigma}_{\mathrm{e}_{14}}}{\tilde{\sigma}_{\mathrm{e}_{1}}^{2}}+\xi_{4} r^{\Delta s_{s}}(n-1) \frac{\tilde{\sigma}_{\mathrm{e}_{15}}}{\tilde{\sigma}_{\mathrm{e}_{1}}^{2}} \\
& +\frac{1}{2} \xi_{1}^{2} r^{2 \Delta s_{2}}(n-1)\left[\frac{\tilde{\sigma}_{\mathrm{e}_{22}}}{\tilde{\sigma}_{\mathrm{e}_{1}}^{2}}+(n-3) \frac{\tilde{\sigma}_{\mathrm{e}_{12}}^{2}}{\tilde{\sigma}_{\mathrm{e}_{1}}^{4}}\right] \\
& \left.+\xi_{1} \xi_{2} r^{\Delta s_{2}+\Delta s_{3}}(n-1)\left[\frac{\tilde{\sigma}_{\mathrm{e}_{23}}}{\tilde{\sigma}_{\mathrm{e}_{1}}^{2}}+(n-3)-\frac{\tilde{\sigma}_{\mathrm{c}_{12}} \tilde{\sigma}_{\mathrm{e}_{13}}}{\tilde{\sigma}_{\mathrm{e}_{1}}^{4}}\right]\right\} .
\end{aligned}
$$

We combine (3.10) and (3.14) with the elastic power-law stress strain relation (3.5) to arrive at the following relations:

$$
\varepsilon_{\beta \lambda}=\varepsilon_{\beta \lambda}^{\mathrm{c}}+\varepsilon_{\beta \lambda}^{\mathrm{p}}
$$

where the elastic strain is given by

$$
\varepsilon_{\beta \lambda}^{\mathrm{e}}=\varepsilon_{0} K_{1} r^{s_{1}}\left(\widetilde{\varepsilon}_{\beta \lambda_{1}}^{\mathrm{e}}+\xi_{1} r^{\Delta s_{2}} \widetilde{\varepsilon}_{\beta \lambda_{2}}^{\mathrm{e}}+\xi_{2} r^{\Delta s_{3}} \widetilde{\varepsilon}_{\beta \lambda_{3}}^{\mathrm{e}}+\xi_{3} r^{\Delta s_{4}} \widetilde{\varepsilon}_{\beta \lambda_{4}}^{\mathrm{e}}+\xi_{4} r^{\Delta s_{5}} \widetilde{\varepsilon}_{\beta \lambda_{5}}^{\mathrm{e}}\right),
$$

with

$$
\tilde{\varepsilon}_{\beta \lambda_{i}}^{\mathrm{e}}=(1+v) \tilde{\sigma}_{\beta \lambda_{i}}+\delta_{\beta \lambda} \Gamma \tilde{\sigma}_{\beta \lambda_{i}}, \quad i=1,2,3,4,5,
$$

and the plastic strain is

$$
\begin{aligned}
\varepsilon_{\beta i}^{\mathrm{p}}= & \frac{3}{2} \alpha \varepsilon_{0}\left(\frac{\sigma_{\mathrm{e}}}{\sigma_{0}}\right)^{n-1} \frac{P_{\beta i \lambda}}{\sigma_{0}} \\
= & \alpha \varepsilon_{0} K_{1}^{n} r^{n s_{1}}\left[\tilde{\varepsilon}_{\beta \lambda_{1}}^{\mathrm{p}} \mid \xi_{1} r^{\Delta s_{2}} \tilde{\varepsilon}_{\beta \lambda_{2}}^{\mathrm{p}}+\xi_{1}^{2} r^{2 \Delta s_{2}} \tilde{\varepsilon}_{\beta \lambda_{22}}^{\mathrm{p}}\right. \\
& \left.+\xi_{2} r^{\Delta s_{3}} \tilde{\varepsilon}_{\beta \lambda_{3}}^{\mathrm{p}}+\xi_{1} \xi_{2} r^{\Delta s_{2}+\Delta s_{3}} \tilde{\varepsilon}_{\beta \lambda_{23}}^{\mathrm{p}}+\xi_{3} r^{\Delta s_{4}} \tilde{\varepsilon}_{\beta \lambda_{4}}^{\mathrm{p}}+\xi_{4} r^{\Delta s_{5}} \tilde{\varepsilon}_{\beta \lambda_{5}}\right]
\end{aligned}
$$

with 


$$
\begin{aligned}
& \tilde{\varepsilon}_{\beta i_{1}}=\frac{3}{2} \tilde{\sigma}_{\mathrm{c}_{1}}^{n-1} \tilde{s}_{\beta i_{1},}, \\
& \tilde{\varepsilon}_{\beta i_{2}}=\frac{3}{2}_{2} \tilde{\sigma}_{\mathrm{c}_{1}}^{n-1}\left[(n-1) \frac{\tilde{\sigma}_{\mathrm{c}_{12}}}{\tilde{\sigma}_{\mathrm{c}_{1}}^{2}} \tilde{s}_{\beta \lambda_{1}}+\tilde{s}_{\beta \lambda_{2}}\right] \text {, }
\end{aligned}
$$

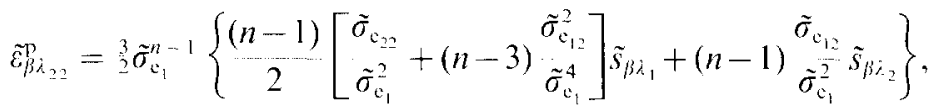

$$
\begin{aligned}
& \tilde{s}_{\beta \lambda_{3}}=\frac{3}{2} \tilde{\sigma}_{c_{1}}^{n-1}\left[(n-1) \frac{\tilde{\sigma}_{c_{13}}}{\tilde{\sigma}_{c_{1}}^{2}} \tilde{s}_{\beta \lambda_{1}}+\tilde{s}_{\beta \lambda_{3}}\right], \\
& \tilde{\sigma}_{\beta i_{4}}=\frac{3}{2} \tilde{\sigma}_{\mathrm{c}_{1}}^{n-1}\left[\begin{array}{ll}
(n-1) & \begin{array}{c}
\tilde{\sigma}_{\mathrm{c}_{14}} \\
\tilde{\sigma}_{\mathrm{c}_{1}}^{2}
\end{array} \tilde{s}_{\beta \lambda_{1}}+\tilde{s}_{\beta \lambda_{4}}
\end{array}\right] \text {, } \\
& \tilde{\varepsilon}_{\beta \lambda_{23}}^{\mathrm{p}}={ }_{2}^{3} \tilde{\sigma}_{\mathrm{c}_{1}}^{n \cdots}(n-1)\left\{\left[\begin{array}{c}
\tilde{\sigma}_{\mathrm{c}_{23}} \\
\hdashline \tilde{\sigma}_{\mathrm{c}_{1}}^{2}
\end{array}\right.\right.
\end{aligned}
$$

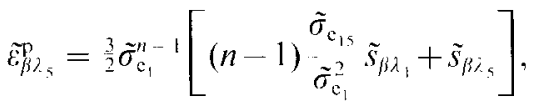

and

$$
\tilde{s}_{\beta \lambda_{i}}=\tilde{\sigma}_{\beta \lambda_{i}}-\frac{1}{2} \tilde{\sigma}_{p p_{i}} \delta_{\beta i \lambda}, \quad i=1,2,3,4,5
$$

\subsection{Governing equation}

The equation governing the stress function is obtained by applying (3.15) to the strain compatibility equation

$$
\frac{1}{r} \frac{\partial^{2}}{\partial r^{2}}\left(r \varepsilon_{\theta}\right)+\frac{1}{r^{2}} \partial \partial^{2} \varepsilon_{r}-\frac{1}{r} \frac{\partial}{\partial r} \varepsilon_{r}-\frac{2}{r^{2}} \frac{\partial^{2}}{\partial r \partial \theta}\left(r \varepsilon_{, \theta}\right)=0 .
$$

The resulting nonlinear eigenvalue equation is

$$
\begin{aligned}
& \alpha K_{1}^{n} r^{n s_{1}} \quad{ }^{2} \Pi_{1}^{\mathrm{p}}+\alpha K_{1}^{n} \xi_{1} r^{n s_{1}+\Delta s_{2}-2} \Pi_{2}^{\mathrm{p}}+\alpha K_{1}^{n} \xi_{1}^{2} r^{n s_{1}+2 \Delta s_{2} \cdot{ }^{2} \Pi_{3}^{\mathrm{p}}} \\
& \quad+\alpha K_{1}^{n} \xi_{2} r^{n s_{1}+\Delta s_{3}-2} \Pi_{4}^{\mathrm{p}}+\alpha K_{1}^{n} \xi_{1} \xi_{2} r^{n s_{1}+\Delta s_{2}+\Delta s_{3}}{ }^{2} \Pi_{5}^{\mathrm{p}} \\
& \quad+\alpha K_{1}^{n} \xi_{3} r^{n s_{1}+\Delta s_{4}}{ }^{2} \Pi_{6}^{\mathrm{p}}+\alpha K_{1}^{n} \xi_{4} r^{n s_{1}+\Delta s_{5}-2} \Pi_{7}^{\mathrm{p}}+K_{1} r^{s_{1} \cdot{ }^{2}} \Pi_{1}^{\mathrm{c}} \\
& \quad+K_{1} \xi_{1} r^{s_{1}+\Delta s_{2}}{ }^{2} \Pi_{2}^{\mathrm{c}}=0,
\end{aligned}
$$

where

$$
\begin{aligned}
& \Pi_{1}^{\mathrm{p}}=\ddot{\tilde{\varepsilon}}_{r_{1}}^{\mathrm{p}}-n s_{1}\left(n s_{1}+2\right) \tilde{\varepsilon}_{r_{1}}^{\mathrm{p}}-2\left(n s_{1}+1\right) \dot{\tilde{\varepsilon}}_{r t_{1}}^{\mathrm{p}_{1}}, \\
& \Pi_{2}^{\mathrm{p}}=\ddot{\tilde{\varepsilon}}_{r_{2}}^{\mathrm{p}}-\left(n s_{1}+\Delta s_{2}\right)\left(n s_{1}+\Delta s_{2}+2\right) \tilde{\varepsilon}_{r_{2}}^{\mathrm{p}}-2\left(n s_{1}+\Delta s_{2}+1\right) \dot{\tilde{\varepsilon}}_{r t_{2}}^{\mathrm{p}}, \\
& \Pi_{3}^{\mathrm{p}}=\ddot{\tilde{\varepsilon}}_{r_{22}}^{\mathrm{p}}-\left(n s_{1}+2 \Delta s_{2}\right)\left(n s_{1}+2 \Delta s_{2}+2\right) \tilde{\varepsilon}_{r_{22}}^{\mathrm{p}}-2\left(n s_{1}+2 \Delta s_{2}+1\right) \dot{\tilde{\varepsilon}}_{r \theta_{22}}^{\mathrm{p}}, \\
& \Pi_{4}^{\mathrm{p}}=\ddot{\tilde{\varepsilon}}_{r_{3}}^{\mathrm{p}}-\left(n s_{1}+\Delta s_{3}\right)\left(n s_{1}+\Delta s_{3}+2\right) \tilde{\varepsilon}_{r_{1}}^{\mathrm{p}}-2\left(n s_{1}+\Delta s_{3}+1\right) \dot{\bar{\varepsilon}}_{r t_{3}}^{\mathrm{p}},
\end{aligned}
$$




$$
\begin{aligned}
& \Pi_{s}^{\mathrm{p}}=\ddot{\tilde{\varepsilon}}_{r_{23}}^{\mathrm{p}}-\left(n s_{1}+\Delta s_{2}+\Delta s_{3}\right)\left(n s_{1}+\Delta s_{2}+\Delta s_{3}+2\right) \tilde{\varepsilon}_{r_{23}}^{\mathrm{p}}-2\left(n s_{1}+\Delta s_{2}+\Delta s_{3}+1\right) \dot{\tilde{\varepsilon}}_{r \theta_{23}}^{\mathrm{p}}, \\
& \Pi_{6}^{\mathrm{p}}=\ddot{\tilde{\varepsilon}}_{r_{4}}^{\mathrm{p}}-\left(n s_{1}+\Delta s_{4}\right)\left(n s_{1}+\Delta s_{4}+2\right) \tilde{\varepsilon}_{r_{4}}^{\mathrm{p}} \quad 2\left(n s_{1}+\Delta s_{4}+1\right) \dot{\tilde{\varepsilon}}_{r \theta_{4}}^{\mathrm{p}}, \\
& \Pi_{7}^{\mathrm{p}}=\ddot{\tilde{\varepsilon}}_{r_{5}}^{\mathrm{p}}-\left(n s_{1}+\Delta s_{5}\right)\left(n s_{1}+\Delta s_{5}+2\right) \tilde{\varepsilon}_{r_{5}}^{\mathrm{p}}-2\left(n s_{1}+\Delta s_{5}+1\right) \dot{\tilde{\varepsilon}}_{r \theta_{5}}^{\mathrm{p}}, \\
& \Pi_{1}^{\mathrm{c}}=\ddot{\tilde{\varepsilon}}_{r_{1}}^{\mathrm{c}}-s_{1} \tilde{\varepsilon}_{r_{1}}^{\mathrm{c}}+s_{1}\left(s_{1}+1\right) \tilde{\varepsilon}_{\theta_{1}}^{\mathrm{e}}-2\left(s_{1}+1\right) \dot{\tilde{\varepsilon}}_{r \theta_{1}}^{\mathrm{e}}, \\
& \Pi_{2}^{\mathrm{c}}=\ddot{\tilde{\varepsilon}}_{r_{2}}^{\mathrm{c}}-\left(s_{1}+\Delta s_{2}\right) \widetilde{\varepsilon}_{r_{2}}^{\mathrm{c}}+\left(s_{1}+\Delta s_{2}\right)\left(s_{1}+\Delta s_{2}+1\right) \tilde{\varepsilon}_{\theta_{2}}^{\mathrm{e}}-2\left(s_{1}+\Delta s_{2}+1\right) \dot{\tilde{\varepsilon}}_{r 0_{2}}^{\mathrm{c}} .
\end{aligned}
$$

Traction free conditions on crack face require:

$$
\tilde{\phi}_{i}(\pi)=\dot{\widehat{\phi}}_{i}(\pi)=0, \quad i=1,2,3,4,5 .
$$

At $\theta=0$, these symmetry conditions are imposed :

$$
\dot{\widetilde{\phi}}_{i}(0)=\ddot{\widetilde{\phi}}_{i}(0)=0, \quad i=1,2,3,4,5 \text {. }
$$

Equations (3.22)-(3.25) comprise the governing equations for the asymptotic expansion.

\section{Solution of Governing Equation}

\subsection{First-order field}

The first-order field is obtained by solving the nonlinear eigenvalue problem associated with the leading term in (3.22)

$$
\begin{aligned}
\Pi_{1}^{\mathrm{p}} & =0, \\
\dot{\widehat{\phi}}_{1}(0) & =\ddot{\widetilde{\phi}}_{1}(0)=0, \\
\widetilde{\phi}_{1}(\pi) & =\dot{\widetilde{\phi}}_{1}(\pi)=0, \\
\max \left\{\tilde{\sigma}_{\mathrm{c}_{1}}\right\} & =1,
\end{aligned}
$$

where $\Pi_{1}^{p}$ is defined by the first equation in (3.23). The solution to this problem is given by HutCHINSON (1968) and RICE and ROSENGREN (1968). The exponent $s_{1}$ of the HRR field can be determined by an application of the $J$-integral (RICE, 1968). The value of $s_{1}$ is

$$
s_{1}=-\frac{1}{n+1} .
$$

The HRR field has been tabulated by Symington et al. (1988).

\subsection{Second-order field}

LI and WANG (1986) were the first to investigate the second order stress field associated with the second term in (3.22), i.e.

$$
\Pi_{2}^{p}=0,
$$


and the associated homogeneous boundary conditions (3.24) and (3.25) for $i=2$. This is a linear eigenvalue problem and they presented the solution using the normalization

$$
\tilde{\phi}_{2}(0)=-1 \text {. }
$$

The second-order stress field also has been studied by Sharma and ARavas (1991). They found that the effects of elasticity enter the solution to second order when $1<n<1.6$. In this case, the second-order field is governed by the following nonhomogeneous linear ordinary differential equation,

$$
\Pi_{2}^{p}=-\Pi_{1}^{c} \text {. }
$$

To obtain the above equation, we have prescribed $\alpha K_{1}^{n} \xi_{1}-K_{1}$ with no loss of generality. The stress exponent of second-order field is given by Curve 4 in Fig. 1(b) when $1<n<1.6$ and by Curve 1 when $n \geqslant 1.6$. Readers are referred to SHARma and ARAvas (1991) for details of the second-order field.

\subsection{Third-order field}

An inspection of (3.22) indicates that one (or more) of three terms can constitute the third-order stress field. These are the third, and fourth and the eighth term in (3.22), i.e.

$$
\begin{aligned}
& \alpha K_{1}^{n} \xi_{1}^{2} r^{n s_{1}+2 \Delta s_{2}-2} \Pi_{3}^{\mathrm{p}}, \\
& \alpha K_{1}^{n} \xi_{2} r^{n s_{1}+\Delta s_{3} \cdot{ }^{2}} \Pi_{4}^{\mathrm{p}}, \\
& K_{1} r^{s_{1}}{ }^{2} \Pi_{1}^{\mathrm{c}} .
\end{aligned}
$$

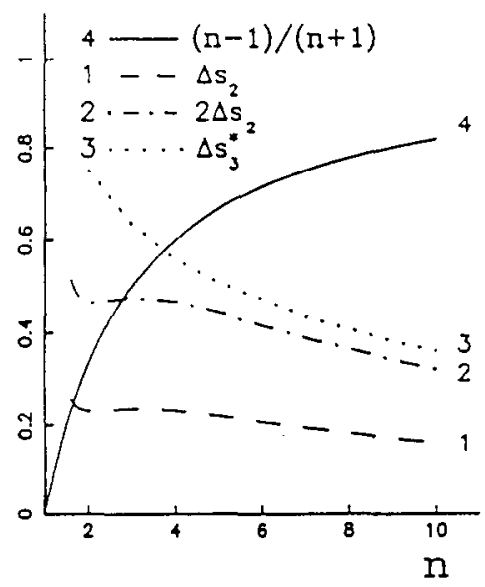

(a)

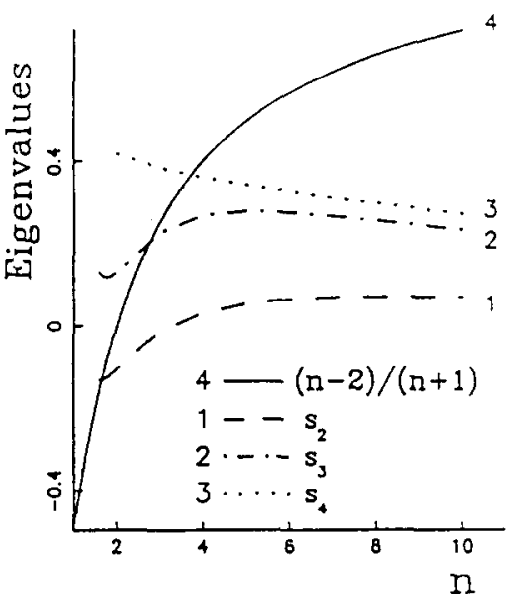

(b)

Fig. I. Eigenvalues for second-, third- and fourth-order stress fields. 
To determine which of these terms make up the third-order stress field, we investigate these exponents,

$$
\begin{aligned}
& n s_{1}+2 \Delta s_{2}-2, \\
& n s_{1}+\Delta s_{3}-2, \\
& s_{1}-2,
\end{aligned}
$$

to find the smallest of the three. This is equivalent to searching for the smallest among the following three numbers:

$$
\begin{aligned}
& 2 \Delta s_{2}, \\
& \Delta s_{3}, \\
& n-1 \\
& n+1
\end{aligned} .
$$

The value of $2 \Delta s_{2}$ is known from the solution for the second-order field. This is shown as Curve 2 in Fig. l(a). i.e.

In order to find the value of $\Delta s_{3}$, we seek the solution to the fourth term of (3.22),

$$
\Pi_{4}^{p}=0,
$$

where $\Pi_{4}^{\mathrm{p}}$ is defined in (3.23). Making use of (3.18), we transform (4.6) into a homogeneous linear ordinary differential equation of the form,

$$
\Pi_{4}^{\mathrm{p}}=D_{1} \dddot{\widetilde{\phi}}_{3}+D_{2} \ddot{\widetilde{\phi}}_{3}+D_{3} \ddot{\widetilde{\phi}}_{3}+D_{4} \dot{\widetilde{\phi}}_{3}+D_{5} \widetilde{\phi}_{3}=0 \text {. }
$$

Here $D_{1}, D_{2}, D_{3}, D_{4}$ and $D_{5}$ are functions of $s_{3}, \Delta s_{3}$ and $\tilde{\phi}_{1}, \dot{\bar{\phi}}_{1}, \dddot{\widetilde{\phi}}_{1}, \dddot{\widetilde{\phi}}_{1}$. The $D$ 's are given in the Appendix.

The eigenvalue $\Delta s_{3}$ (or $s_{3}$ ) can be determined in the following manner. Since (4.7) is a linear equation for $\tilde{\phi}_{3}(0)$, we first seek the two elementary solutions which satisfy these conditions :

$$
\begin{array}{lll}
\text { (1) } & \tilde{\phi}_{3}(0)=1, & \ddot{\tilde{\phi}}_{3}(0)=0, \\
\text { (2) } & \tilde{\phi}_{3}(0)=0, & \ddot{\tilde{\phi}}_{3}(0)=1,
\end{array}
$$

respectively. Designate the elementary solutions corresponding to the initial conditions-(4.8) and (3.25) $(i=3)$, and (4.9) and (3.25) $(i=3)-$ by $\tilde{\phi}_{3}^{(1)}(\theta)$ and $\widetilde{\phi}_{3}^{(2)}(\theta)$, respectively. The integration of (4.7) is performed by the Runge-Kutta method with automatic step-size control; the integration error is kept to within $10^{-8}$.

The general solution to (4.7) has the form

$$
\widetilde{\phi}_{3}(\theta)=C_{1} \widetilde{\phi}_{3}^{(1)}(\theta)+C_{2} \widetilde{\phi}_{3}^{(2)}(\theta) .
$$

Satisfaction of boundary conditions at $\theta=\pi,(3.24)(i=3)$, requires

$$
\begin{aligned}
& C_{1} \tilde{\phi}_{3}^{(1)}(\pi)+C_{2} \widetilde{\phi}_{3}^{(2)}(\pi)=0, \\
& C_{1} \dot{\widetilde{\phi}}_{3}^{(1)}(\pi)+C_{2} \dot{\widetilde{\phi}}_{3}^{(2)}(\pi)=0 .
\end{aligned}
$$


A non-trivial solution for $C_{1}$ and $C_{2}$ exists if and only if the determinant of the matrix of the coefficients vanishes, i.e.

$$
\widetilde{\phi}_{3}^{(1)}(\pi) \dot{\vec{\phi}}_{3}^{(2)}(\pi)-\widetilde{\phi}_{3}^{(2)}(\pi) \dot{\widetilde{\phi}}_{3}^{(1)}(\pi)=0
$$

The solution to (4.12) provides the value of $\Delta s_{3}$.

In general two different values of $\Delta s_{3}$ are obtained for a given value of $n$. It is found that equation (4.6) is similar to (4.3), so that the smaller value of $\Delta s_{3}$ is the same as the value of $\Delta s_{2}$. The second (larger) value of $\Lambda s_{3}$ is denoted by $\Lambda s_{3}^{*}$

The values of $\Delta s_{2}, 2 \Delta s_{2}, \Delta s_{3}^{*}$ and $(n-1) /(n+1)$ are plotted in Fig. 1(a) for $1<n \leqslant 10$. It can be seen that the stress exponent of the third-order field is given by Curve 4 when $1.6<n \leqslant 2.8$, i.e. $\Delta s_{3}=(n-1) /(n+1)$. It is given by Curve 2 when $n>2.8$, i.e. $\Delta s_{3}=2 \Delta s_{2}$.

It is apparent from the above discussions that the equation governing the thirdorder field depends on the value of $n$.

Case $1: 1.6<n \leqslant 2.8$

The governing equation is

$$
\Pi_{4}^{p}=D_{1} \dddot{\widetilde{\phi}}_{3}+D_{2} \ddot{\widetilde{\phi}}_{3}+D_{3} \ddot{\widetilde{\phi}}_{3}+D_{4} \dot{\widetilde{\phi}}_{3}+D_{5} \widetilde{\phi}_{3}=-\Pi_{1}^{\mathrm{c}},
$$

and $\alpha K_{1}^{\prime \prime} \xi_{2}=K_{1}$ has been used in the above. As discussed previously, $\Pi_{1}^{\mathrm{c}}$ is related to the first-order field so that $\Delta s_{3}=(n-1) /(n+1)$. That is, the stress exponent $s_{3}$ of the third-order field is equal to $(n-2) /(n+1)$ which is shown as Curve 4 in Fig. 1(b).

Case $2: 2.8<n \leqslant 15 \dagger$

The governing equation is

$$
\Pi_{4}^{\mathrm{p}}=D_{1} \dddot{\widetilde{\phi}}_{3}+D_{2} \dddot{\widetilde{\phi}}_{3}+D_{3} \ddot{\widetilde{\phi}}_{3}+D_{4} \dot{\tilde{\phi}}_{3}+D_{5} \tilde{\phi}_{3}=-\Pi_{3}^{\mathrm{p}},
$$

The relationship $\xi_{2}=\xi_{1}^{2}$ has been used in (4.14) and $\Pi_{3}^{p}$ is related to the first-and second-order fields. In this case, $\Delta s_{3}=2 \Delta s_{2}$, so that the stress exponent $s_{3}$ is equal to $2 s_{2}-s_{1}$.

Let $\tilde{\phi}_{3}^{(0)}(\theta)$ be a particular solution that satisfies (4.13), or (4.14), and also meets the homogeneous initial conditions,

$$
\widetilde{\phi}_{3}(0)=\dot{\widetilde{\phi}}_{3}(0)=\ddot{\widetilde{\phi}}_{3}(0)=\ddot{\widetilde{\phi}}_{3}(0)=0 .
$$

Combining $\tilde{\phi}_{3}^{(0)}(\theta)$ with the solution in (4.10) provides the general solution for (4.13). or (4.14),

$$
\tilde{\phi}_{3}=C_{1} \tilde{\phi}_{3}^{(1)}+C_{2} \tilde{\phi}_{3}^{(2)}+\tilde{\phi}_{3}^{(0)} .
$$

Now the boundary conditions in (3.24) are invoked to determine the coefficients $C_{1}$ and $C_{2}$. The outcome is:

$$
\begin{aligned}
& C_{1} \widetilde{\phi}_{3}^{(1)}(\pi)+C_{2} \widetilde{\phi}_{3}^{(2)}(\pi)=-\widetilde{\phi}_{3}^{(0)}(\pi) \\
& C_{1} \dot{\widetilde{\phi}}_{3}^{(1)}(\pi)+C_{2} \dot{\widetilde{\phi}}_{3}^{(2)}(\pi)=-\dot{\widetilde{\phi}}_{3}^{(0)}(\pi)
\end{aligned}
$$

$\uparrow$ We did not perform calculations for $n>15$ since it is known that the robustness of such asymptotic expansions deteriorates as $n$ becomes large. Moreover, detailed numerical descriptions of complete ficlds for the purpose of "field matching" are available only for $n \leqslant 10$. 
A unique solution for $C_{1}$ and $C_{2}$ exists since the determinant in (4.17) does not vanish in general.

In summary, the third-order stress field is not independent over the range of hardening values of interest. The third-order field is controlled by the amplitudes of the lower-order fields: $K_{3}=1 /\left(\alpha K_{1}^{n-2}\right)$ for $1.6<n \leqslant 2.8, \quad K_{3}=K_{2}^{2} / K_{1}$ for $2.8<n \leqslant 15$.

\subsection{Higher-order fields}

The analysis of the fourth- and fifth-order fields proceeds in the same manner. This will not be discussed in the interest of space. Figue $1(\mathrm{~b})$ shows how the stress exponents of the fields of different order depend on $n$. For $2.8<n \leqslant 3.7$, the stress exponent of the fourth-order field is given by Curve 4 . For $n>3.7$ it is given by Curve 3 . The eigenfields for $n=3,5,7$ and 10 are discussed in the next section.

\section{Results}

The analysis of higher-order fields has been carried out for $1<n \leqslant 15$. Eigenfields for $n=3,5,7$ and 10 and comparisons with full-field numerical solutions obtained by modified boundary layer analyses are discussed here.

\subsection{Solutions for $n=3$}

The first- to the fifth-order stress fields have been obtained for $n=3 ; \theta$-variations of the eigenfields are shown in Fig. 2. The stress solution can be written in the form:

$$
\begin{aligned}
& \frac{\sigma_{\beta i}}{\sigma_{0}}=\sum_{i=1}^{5} K_{i} r^{s} \tilde{\sigma}_{\beta \lambda_{i}}(\theta) \\
& =K_{1} r^{-1 /(n+1)} \tilde{\sigma}_{\beta \lambda_{1}}(\theta)+K_{2} r^{s_{2}} \tilde{\sigma}_{\beta \lambda_{2}}(\theta) \\
& +\frac{K_{2}^{2}}{K_{1}} r^{2 s_{2}+1 /(n+1)} \tilde{\sigma}_{\beta \hat{\beta}_{3}}(\theta)+\frac{1}{\alpha K_{1}^{n-2}} r^{(n-2) /(n+1)} \tilde{\sigma}_{\beta \lambda_{4}}(\theta)+K_{5} r^{s_{5}} \tilde{\sigma}_{\beta \lambda_{5}}(\theta) \\
& =\left(\frac{J}{\alpha \varepsilon_{0} \sigma_{0}} \bar{I}_{n} r\right)^{0.25} \tilde{\sigma}_{\beta \lambda_{1}}(\theta)+K_{2} r^{-0.01284028} \tilde{\sigma}_{\beta \lambda_{2}}(\theta) \\
& +\left(\frac{\alpha \varepsilon_{0} \sigma_{0} I_{n}}{J}\right)^{0.25} K_{2}^{2} r^{0.22431944} \tilde{\sigma}_{\beta \gamma_{3}}(\theta)+\frac{1}{\alpha}\left(\frac{\alpha \varepsilon_{0} \sigma_{0} I_{n}}{J}\right)^{0.25} r^{0.25} \tilde{\sigma}_{\beta \lambda_{4}}(\theta) \\
& +K_{5} r^{0.38221612} \tilde{\sigma}_{\beta \lambda,}(\theta) \text {. }
\end{aligned}
$$

Note that elasticity effects enter the fourth-order field which is evaluated for $v=0.5$. The fifth-order stress field is normalized by requiring $\bar{\phi}_{5}(0)=-1$. In writing (5.1), the relationships $K_{3}=K_{2}^{2} / K_{1}$ and $K_{4}=1 /\left(\alpha K_{1}^{n-2}\right)$ have been used. That is, among the five amplitude coefficients, $K_{i}(i=1,2,3,4,5)$, only three can be independently prescribed. They are $K_{1}, K_{2}$ and $K_{5}$. 


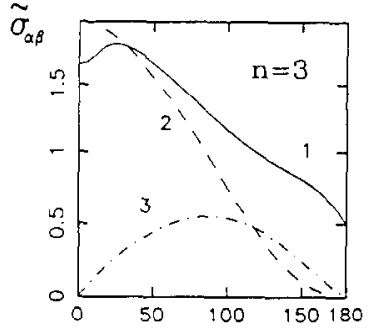

(a)

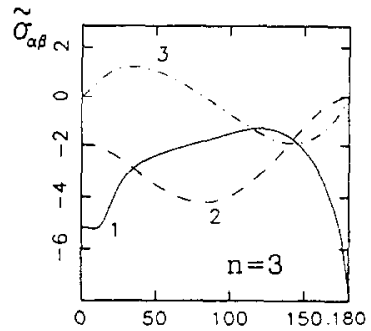

(b) $\theta$

the 2nd field

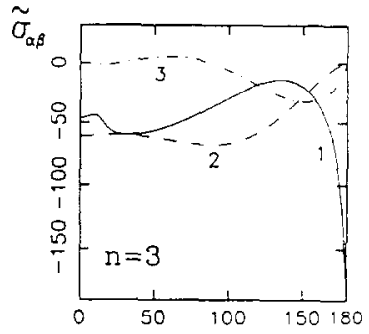

(c)

the 1st field

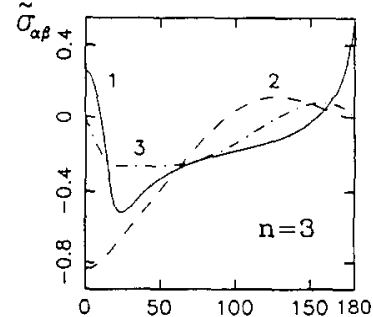

(d)

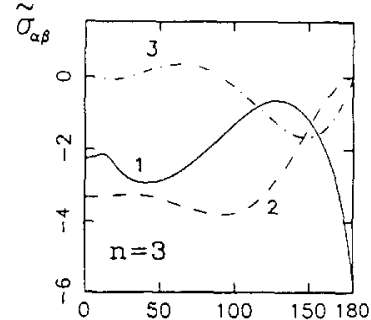

(e)

the 4 th field

Fig. 2. Angular distributions of the first- to fifth-order stress lields for $n=3 . \tilde{\sigma}_{r_{i}}$ is identified by label 1 , $\tilde{\sigma}_{\theta}$ by label 2 , and $\tilde{\tau}_{r \theta}$ by label 3 .

\subsection{Solutions for $\mathrm{n}=5,7$ and 10}

Figure 3 shows the angular distributions of the first- to fourth-order stress fields for $n=5,7$ and 10. It can be seen that the patterns of the first-, second-and fourthorder stress fields for $n=5$ are similar to the corresponding stress patterns for $n=7$ and 10. By contrast, the pattern of the third-order stress field depends sensitively on $n$. Collectively, the stress fields can be written in the form :

$$
\begin{aligned}
\frac{\sigma_{\beta \lambda}}{\sigma_{0}}= & \sum_{i=1}^{4} K_{i} r^{s_{i}} \tilde{\sigma}_{\beta \lambda_{i}}(\theta) \\
= & K_{1} r^{-1 /(n+1)} \tilde{\sigma}_{\beta i \lambda_{1}}(\theta)+K_{2} r^{s_{2}} \tilde{\sigma}_{\beta \lambda_{2}}(\theta)+\frac{K_{2}^{2}}{K_{1}^{2}} r^{2 s_{2}+1 /(n+1)} \tilde{\sigma}_{\beta \lambda_{3}}(\theta)+K_{4} r^{s_{4}} \tilde{\sigma}_{\beta \lambda_{4}}(\theta) \\
= & \left(\frac{J}{\alpha \varepsilon_{0}} \frac{J}{\sigma_{0} I_{n} r}\right)^{1 /(n+1)} \tilde{\sigma}_{\beta \lambda_{1}}(\theta)+K_{2} r^{s_{2}} \tilde{\sigma}_{\beta \lambda_{2}}(\theta) \\
& +\left(\frac{\alpha \varepsilon_{0} \sigma_{0} I_{n}}{J}\right)^{1 /(n+1)} K_{2}^{2} r^{s_{3}} \tilde{\sigma}_{\beta \lambda_{3}}(\theta)+K_{4} r^{s_{4}} \tilde{\sigma}_{\beta \lambda_{4}}(\theta) .
\end{aligned}
$$




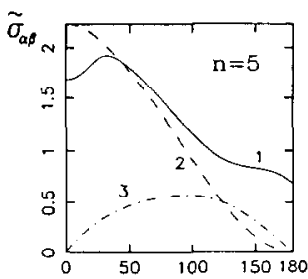

(a)

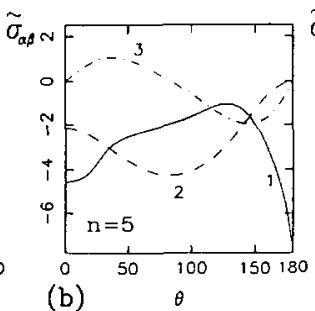

(b)

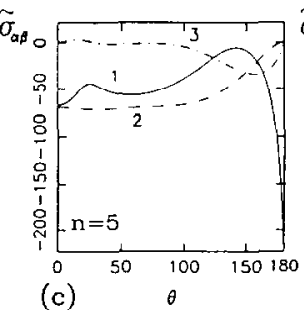

(c)

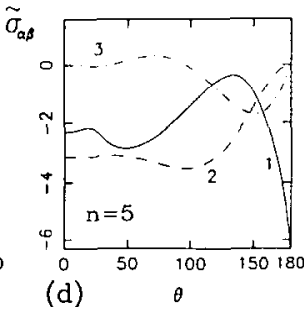

(d)

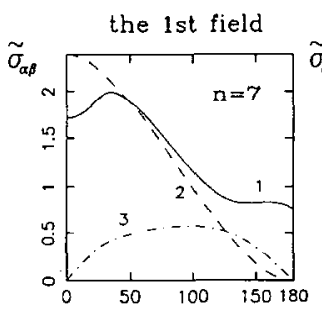

(e)

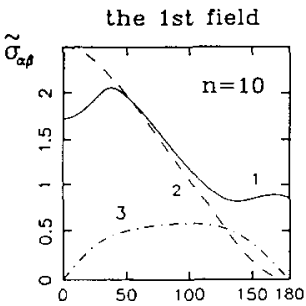

(i)

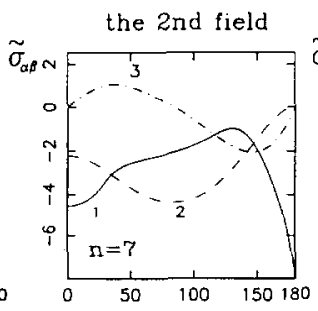

(f)

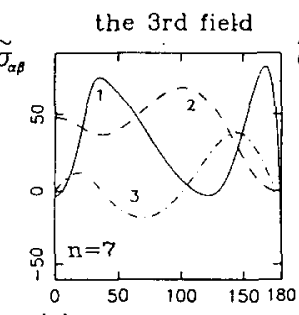

(g)

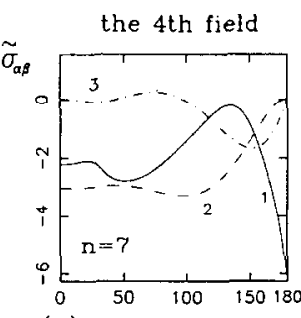

(h)

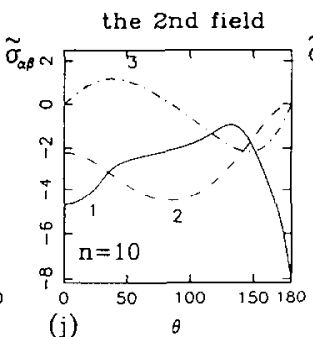

the 2nd field

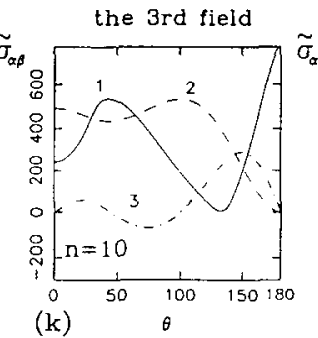

the $3 r d$ field

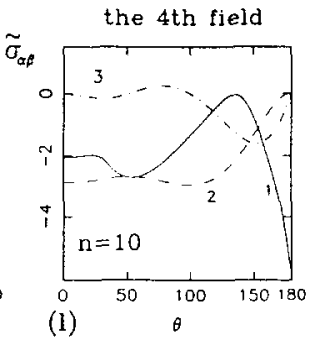

the 4 th field

Fig. 3. Angular distributions of the first- to fourth-order stress fields for $n=5,7$ and 10 . $\tilde{\sigma}_{r_{i}}$ is identified by label $1, \tilde{\sigma}_{\theta_{i}}$ by label 2 , and $\tilde{\tau}_{r l}$ by label 3 .

The fourth-order field is normalized by requiring $\tilde{\phi}_{4}(0)=-1$ and the relationship $K_{3}=K_{2}^{2} / K_{1}$ has been used in (5.2). Therefore, the amplitudes which can be independently prescribed are $K_{1}, K_{2}$ and $K_{4}$. Stress exponents for the first- to fourth-order stress field for $n=3,5,7$ and 10 are given in Table 1 .

\subsection{Comparisons with full-field stress solutions-difference fields}

Figure 4 shows the difference stress fields for $n=10$ corresponding to six different triaxiality levels. These fields were obtained by using the MBL formulation and finite element calculations based on a geometrically rigorous formulation which takes full account of crack tip blunting (O’DOWD and SHIH, 1991). The difference field is introduced in Section 2.2 and defined by (2.5).

To facilitate the comparison with the difference field established by finite element analysis, we write the sum of our second-, third- and fourth-order stress fields for $n=10$ as : 
TABLE 1

\begin{tabular}{lrrrr}
\hline & \multicolumn{1}{c}{$s_{1}$} & \multicolumn{1}{c}{$s_{2}$} & \multicolumn{1}{c}{$s_{3}$} & \multicolumn{1}{c}{$s_{4}$} \\
\hline$n=3$ & -0.25000000 & -0.01284028 & 0.22431944 & 0.25000000 \\
$n=5$ & -0.16666667 & 0.05455957 & 0.27578581 & 0.34072164 \\
$n=7$ & -0.12500000 & 0.06937479 & 0.26374958 & 0.30845004 \\
$n=10$ & -0.09090909 & 0.06976616 & 0.23044141 & 0.26959457 \\
\hline
\end{tabular}

$$
\begin{aligned}
& \left(\begin{array}{c}
\sigma_{\beta i} \\
\sigma_{0}
\end{array}\right)_{\mathrm{diff}}=\sum_{i=2}^{4} K_{i} r_{i} \tilde{\sigma}_{\beta \lambda_{i}}(\theta) \\
& =K_{1} \xi_{1} r^{s_{2}} \tilde{\sigma}_{\beta \lambda_{2}}(\theta)+K_{1} \xi_{1}^{2} r^{2 v_{2}+1:(n+1)} \tilde{\sigma}_{\beta \lambda_{3}}(\theta)+K_{1} \xi_{3} r^{s_{4}} \tilde{\sigma}_{\beta \lambda_{4}}(\theta) \\
& =k_{2}\left(\frac{r}{J / \sigma_{0}}\right)^{0.06976616} \tilde{\sigma}_{\beta i, 2}(\theta)+k_{3}\left(\begin{array}{c}
r \\
J / \sigma_{0}
\end{array}\right)^{0.23044141} \tilde{\sigma}_{B i_{3}}(\theta) \\
& +k_{4}\left(\begin{array}{c}
r \\
J / \sigma_{0}
\end{array}\right)^{0.26959457} \tilde{\sigma}_{\beta \lambda_{4}}(\theta) \text {, }
\end{aligned}
$$

where

$$
k_{2}=K_{1} \xi_{1}\left(\begin{array}{c}
J \\
\sigma_{0}
\end{array}\right)^{3}, \quad k_{3}=K_{1} \xi_{1}^{2}\left(\frac{J}{\sigma_{0}}\right)^{2 s_{2}-s_{1}}, \quad k_{4}=K_{1} \xi_{3}\left(\frac{J}{\sigma_{0}}\right)^{s_{4}},
$$

and

$$
K_{1}=\left(\begin{array}{c}
J \\
\alpha \varepsilon_{0} \sigma_{0} I_{n}
\end{array}\right)^{1:(n+1)}
$$

It is easily shown that

$$
k_{3}=\left(\alpha \varepsilon_{0} I_{n}\right)^{1:(n+1)} k_{2}^{2} .
$$

To carry out the comparisons with the finite element solutions, we take $\alpha=1.0$, $\varepsilon_{0}=1 / 300$, and $I_{n}=4.54$. These are the material constants employed by O'DowD and SHIH (1991).

In the above expression for $\left(\sigma_{\beta i} / \sigma_{0}\right)_{\text {diff }}$ only two coefficients $-k_{2}$ and $k_{4}-$-can be independently prescribed. The amplitudes, $k_{2}$ and $k_{4}$, can be determined by matching the right-hand side of (5.3) with the difference field presented in Fig, 4 at any suitable location. In this study, the "point matching" is enforced at $r /\left(J / \sigma_{0}\right)=2$ and $\theta=0$. Direct attention to one of six angular stress distributions shown in Fig. 4(c) for $r /\left(J / \sigma_{0}\right)=2$. The numerical value of $\left.\left(\sigma_{\nu 0} / \sigma_{0}\right)\right)_{\text {diff }}$ at $\theta=0$ is equated with the linear combination of $k_{2}$ and $k_{4}$ for $\left(\sigma_{\theta \theta} / \sigma_{0}\right)_{\text {diff }}$ from (5.3). Similarly, we equate the corresponding numerical value of $\left(\sigma_{r r} / \sigma_{0}\right)_{\text {dif }}$ in Fig. $4(\mathrm{~d})$ with the linear combination of $k_{2}$ and $k_{4}$ for $\left(\sigma_{r r} / \sigma_{0}\right)_{\text {diff. }}$. This provides two equations for $k_{2}$ and $k_{4}$. The procedure is repeated for the other five distributions presented in Figs $4(\mathrm{c})$ and $4(\mathrm{~d})$. Table 2 lists the values of $\left(\sigma_{\varphi \theta} / \sigma_{0}\right)_{\text {diff }}$ and $\left(\sigma_{r r} / \sigma_{0}\right)_{\text {dirf }}$, corresponding to the six MBL loadings 

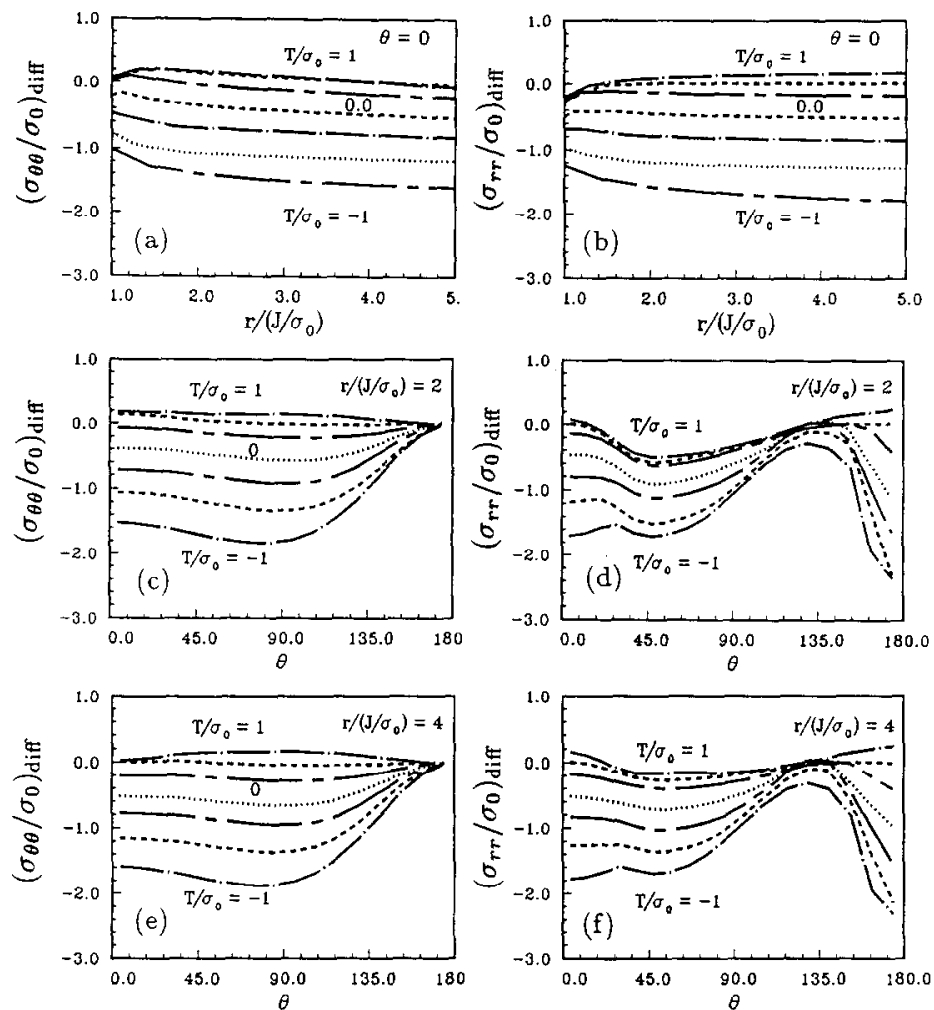

Fig. 4. Difference field from finite element analysis for $n=10$ [Fig. 5 of O'DowD and SHiH (1991)]. Distributions corresponding to six MBL loadings are shown. (a) and (b) Radial distribution of normal stresses at $0=0$. (c) and (d) Angular distribution at $r /\left(J / \sigma_{0}\right)=2$. (e) and (f) Angular distribution at $r /\left(J / \sigma_{0}\right)=4$.

presented in Figs 4(c) and 4(d), and the values of $k_{2}$ and $k_{4}$ obtained by matching; $k_{3}$ is calculated from (5.6).

Figures 5(a)-5(f) show the fields constructed from (5.3) for the values of $k_{2}, k_{3}$ and $k_{4}$ listed in Table 2 . It can be seen that both the radial and the angular distributions of $\left(\sigma_{t t r} / \sigma_{0}\right)_{\mathrm{ditf}}$ and $\left(\sigma_{r} / \sigma_{0}\right)_{\mathrm{d} H \mathrm{H}}$, for all six choices of $k_{2}$ and $k_{4}$, agree well with the finite

TABLE 2. $n=10$

\begin{tabular}{rrrrrr}
\hline & $\left(\sigma_{\theta \theta}\right)_{\mathrm{diff}} / \sigma_{0}$ & $\left(\sigma_{r r}\right)_{\mathrm{diff}} / \sigma_{0}$ & $k_{2}$ & $k_{3}$ & \multicolumn{1}{c}{$k_{4}$} \\
\hline 1 & -1.50 & -1.70 & 0.06844 & 0.00320 & 0.91558 \\
2 & -1.06 & -1.20 & 0.05513 & 0.00208 & 0.61191 \\
3 & -0.74 & -0.80 & 0.04027 & 0.00111 & 0.36945 \\
4 & -0.40 & -0.48 & 0.03212 & 0.00071 & 0.21034 \\
5 & -0.08 & -0.13 & 0.01571 & 0.00017 & 0.04041 \\
6 & 0.20 & 0.10 & 0.01035 & 0.00007 & -0.05275 \\
\hline
\end{tabular}



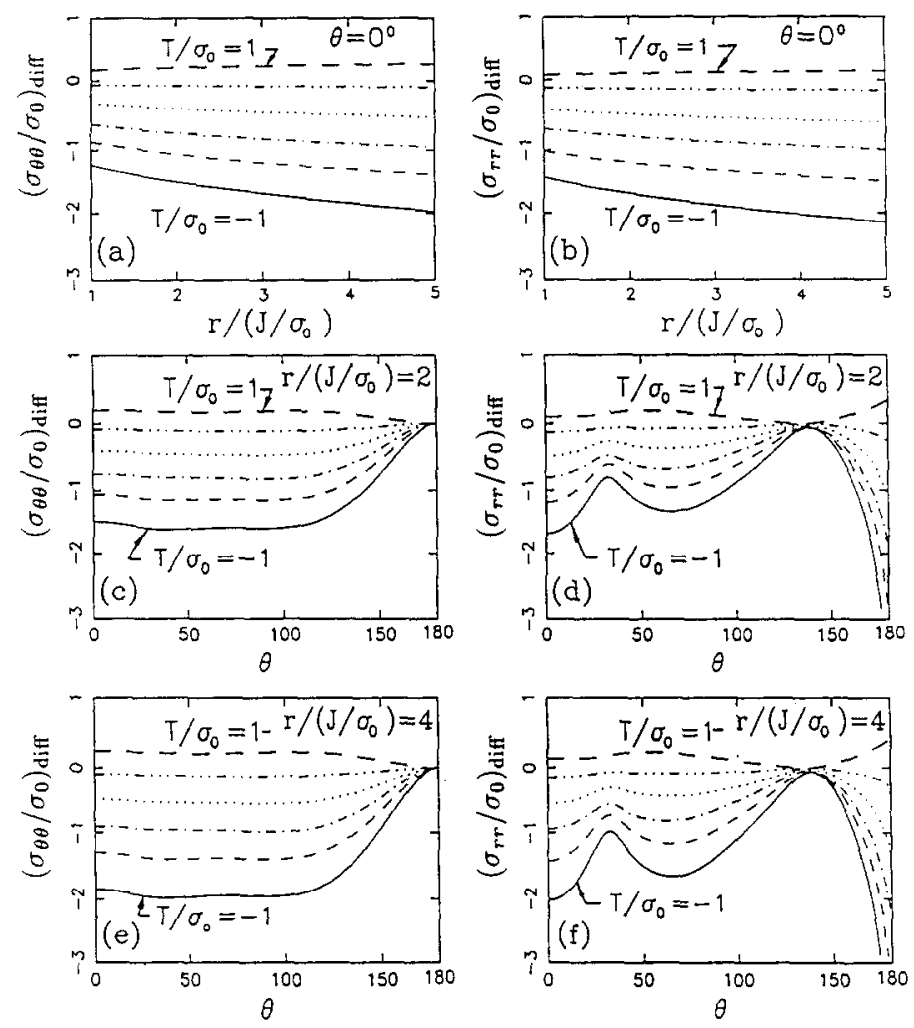

FIG. 5. The sum of the second- to the fourth-order stress fields for $n=10$. Distributions corresponding to six MBL loadings are shown. (a) and (b) Radial distribution of normal stresses at $\theta=0$. (c) and (d) Angular distribution at $r /\left(\delta / \sigma_{0}\right)=2$. (e) and (f) Angular distribution at $r /\left(J / \sigma_{0}\right)=4$.

element solutions shown in Figs 4(a) 4(f). We should add that, though $k_{2}$ and $k_{4}$ were determined by "point matching", the full agreement between the fields in Figs 4 and 5 demonstrates that "field matching" has been achieved.

The shear stresses have not been used in the determination of $k_{2}$ and $k_{4}$. Therefore, they provide an independent check of the quality of agreement (or lack of agreement) between the asymptotic series and complete descriptions of the near-tip fields. The angular distributions of the shear stress, based on the series in (5.2), are shown in Figs 6(a) and 6(b). The solid line is the first-order shear stress, i.e. the HRR field itself, while the non-solid lines are the first-order plus the higher-order shear fields for the six cases listed in Table 2. Now compare the six angular distributions in Figs 6(a) and 6(b), for $r /\left(J / \sigma_{0}\right)=2$ and 4 , with the fields obtained from finite element calculations displayed in Figs 6(c) and 6(d)-the agreement is remarkably good. Figures 6(a) and 6(b) also reveal that the higher-order shear terms, $\left(\sigma_{r+1}\right)_{\text {diff }}$, contribute negligibly to the shear stress in the forward sector, $-\pi / 2<0<\pi / 2$. By contrast, the magnitude of $\left(\sigma_{\theta \theta}\right)_{\text {diti }}$ and $\left(\sigma_{r r}\right)_{\text {diff }}$ can be comparable to $\sigma_{0}$. That is, $\left|\left(\sigma_{\theta \theta}\right)_{\text {diff }}\right| \approx\left|\left(\sigma_{r r}\right)_{\text {dirf }}\right| \gg\left|\left(\sigma_{r \theta}\right)_{\text {difr }}\right|$. This implies that the higher-order terms collectively describe what is essentially a hydrostatic stress field. 

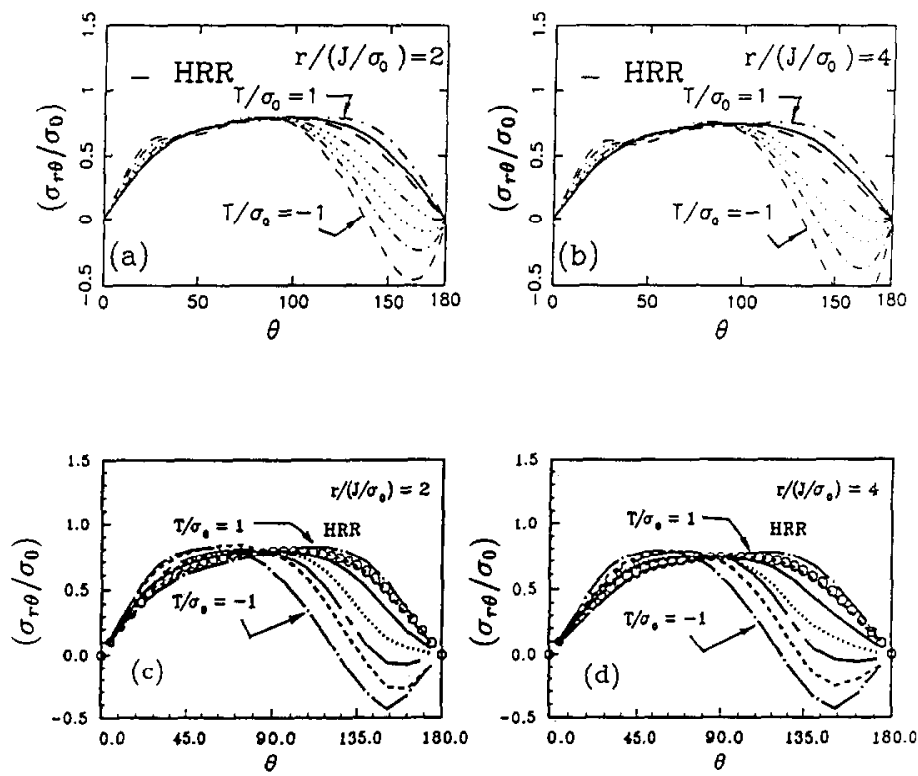

FIG. 6. Angular distributions of shear stress at $r /\left(J / \sigma_{0}\right)=2$ and 4 for $n=10$. Distributions corresponding to six MBL loadings are shown. (a) and (b) Sum of the first- to the fourth-order stress fields are shown by the non-solid lines. The solid line is the HRR field. (c) and (d) Fields from finite element analysis; the open symbols are associated with the HRR field [Fig. 3 of O'DowD and SHIH ( 1991)].

\section{Cleavage Toughness Locus}

It is shown in the preceding section that the hoop stress ahead of the crack tip is adequately represented in terms of $J$ and $Q$ :

$$
\frac{\sigma_{22}}{\sigma_{0}}=\left(\frac{J}{\alpha \varepsilon_{0} \sigma_{0} I_{n} r}\right)^{1 /(n+1)} \tilde{\sigma}_{22}(\theta=0)+Q .
$$

The distribution of the normal stress ahead of cracks in finite width geometries also can be expected to obey the form in (6.1) as long as the characteristic crack dimensions is much larger than $J / \sigma_{0}$. Readers are referred to articles by O'DowD and SHIH (1991, $1992 \mathrm{a}, 1992 \mathrm{~b}$ ) for details. A consequence of a two-parameter theory is this: toughness, $J_{c}$, depends on crack tip stress triaxiality or in this case $Q$. This is explored below.

We impose the condition that the initiation of cleavage fracture requires achieving a critical normal stress, $\sigma_{22}=\sigma_{\mathrm{c}}$, at a critical microstructural distance, $r=r_{\mathrm{c}}$, ahead of the crack tip (RITCHIE et al., 1973). With $J_{\mathrm{c}}^{*}$ and $Q^{*}$ denoting the toughness and crack tip triaxiality associated with a long crack $\left(a / r_{\mathrm{c}}, a / r_{\mathrm{p}} \rightarrow \infty\right)$, or when the remote loading on the crack is described by $K$ alone $(T=0)$, one finds from (6.1) that

$$
\frac{J_{\mathrm{c}}}{J_{\mathrm{c}}^{*}}=\left(\frac{\frac{\sigma_{\mathrm{c}}}{\sigma_{0}}-Q}{\frac{\sigma_{\mathrm{c}}}{\sigma_{0}}-Q^{*}}\right)^{n+1} \approx\left(1-\frac{Q \sigma_{0}}{\sigma_{\mathrm{c}}}\right)^{n+1}
$$


since $Q^{*} \approx 0$. The cleavage toughness, $J_{c}$, is predicted to depend sensitively on $Q$ when $n$ is greater than about 3 . Note that the ratio, $J_{c} / J_{c}^{*}$, does not depend on $r_{\mathrm{c}}$. Further discussions on constraint and size effects on toughness can be found in O’Dowd and SHIH (1992a).

KIRK et al. (1991) have obtained cleavage toughness for A515 steels at room temperature. They tested edge-cracked bend bars with thicknesses $B=10,25.4$ and $50.8 \mathrm{~mm}$ and various crack length to width ratios. $J$ at fracture was evaluated from the load-displacement record and $Q$ at fracture was determined from finite element analyses of the specimens. The experimentally determined toughness locus, $J_{c}$ as a

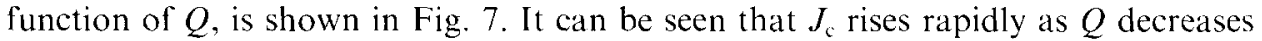
corresponding to a loss of stress triaxiality. Though the trends are similar, the toughness data for the thicker specimens lie above those for the thinnest specimens suggesting a possible thickness effect. The thickness effect could be caused, in large part, by the data reduction for $J$ and $Q$ which was performed with plane strain analysis. We have not ascertained the error introduced by using plane strain solutions for $J$ and $Q$ to interpret the test data obtained from the thinner specimens. Studies aimed at describing the stress-triaxiality near a three-dimensional crack front in thin and thick plates are in progress. For the present, we apply the relation in (6.2) to the data in Fig. 7. The predicted variation of $J_{\mathrm{c}}$ with $Q$ from (6.2) is shown by the dashed line; it captures the trend of the experimental toughness data. The predicted curve is based on $\sigma_{\mathrm{c}}=3.5 \sigma_{0}, J_{\mathrm{c}}^{*}=40 \mathrm{kPa} \cdot \mathrm{m}$ and $n=5$, representative of the strain hardening of A515 steel.

\section{Conclusions}

An analysis of higher-order asymptotic fields, within a small displacement gradient framework, has been carried out for plane strain, Mode I crack in an elastic powerlaw hardening material. A five-term eigen-expansion of the asymptotic stress fields is derived for $n=3$; a four-term expansion is derived for $n=5,7$ and 10 . Our results

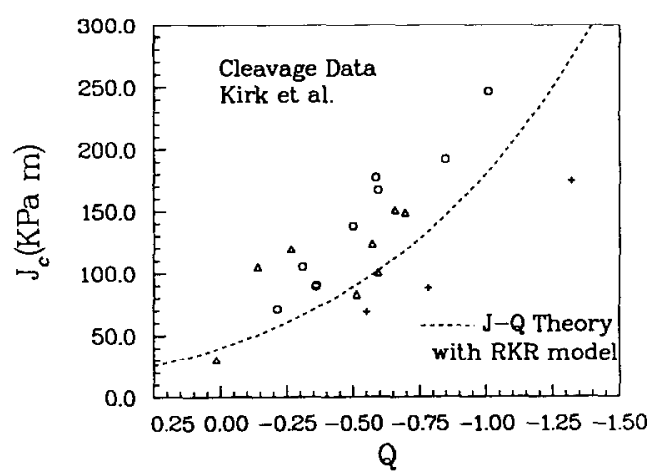

Fig. 7. Cleavage toughness data for ASTM A515 Grade 70 steels tested at $20 \mathrm{C}$ using edge-cracked bend bar for three thicknesses; + for $B=10 \mathrm{~mm}, O$ for $B=25.4 \mathrm{~mm}, \triangle$ for $B=50.8 \mathrm{~mm}$ (KIRK et al., 199l). Toughness curve predicted by (6.2) is indicated by the dashed line. 
show that the material's strain hardening capacity determines the extent to which the elasticity affects the field. For $1.6<n \leqslant 2.8$, elasticity effects enter the third-order stress field. For $2.8<n \leqslant 3.7$, elasticity effects enter the solution to fourth-order. The amplitude of the third-order stress field, $K_{3}$, scales with the amplitude of the secondorder stress field, $K_{2}$, when $n>2.8$. By contrast, the amplitude of the fourth-order stress field, $K_{4}$, is an independent coefficient for $n>3.7$; however, $K_{4}$ scales with $K_{1}$ for $n<3.7$. For $n=3$, the amplitudes that can be independently precribed in the fiveterm series are $K_{1}, K_{2}$ and $K_{5}$. For $n=5,7$ and 10, the independent amplitudes in the four-term series are $K_{1}, K_{2}$ and $K_{4}$.

A two-term expansion for a Mode II crack has been performed by XIA and WANG (1992). Similar two-term expansions for a plane stress, Mode I crack have also been carried out, Li (1988), Sharma and Aravas (1991) and Li and WANG (1992). Readers are referred to these papers for details. A higher-order analysis for Mode I and II cracks has been attempted by CHAO et al. (1991). They utilize the higher-order terms to establish the size and shape of the zone dominated by the HRR field but include little discussion on the character or collective behavior of the higher-order fields.

Through finite element analysis which takes full account of crack tip blunting, O'DowD and SHIH $(1991,1992 a)$ have obtained detailed descriptions of the stress fields surrounding the finite strain zone for $n=10$, representative of moderate to low hardening materials ; difference fields for a wide range of MBL loadings are presented in their paper. Remarkably, the full range of difference fields in O'DowD and SHIH can be reproduced by the present higher-order series by appropriately varying the values of $K_{2}$ and $K_{4}$. The agreement between them persists over distances $1 \leqslant r /\left(J / \sigma_{0}\right) \leqslant 5$, or distances ranging from 2 to 10 crack openings ahead of the crack tip.

The findings pertaining to moderate to low hardening materials are summarized below.

1. The zone of dominance of the four-term asymptotic series extends over microstructurally significant length scales.

2. The four-term series accurately match the complete fields generated by MBL analyses over the full range of loadings.

3. Appropriate choices of $K_{2}$ and $K_{4}$ can generate near-tip fields representative of a wide range of crack tip constraints. Moreover, the higher-order terms collectively describe a spatially uniform hydrostatic stress of adjustable magnitude.

1. The present results support a suggestion that $J$ and a near-tip stress triaxiality parameter can describe the full range of near-tip states surrounding the fracture process zone.

\section{ACKNOWLEDGEMENTS}

LX and TCW are supported by a grant from the National Natural Science Foundation of China. CFS is supported by a grant from the U.S. Nuclear Regulatory Commission.

\section{ReFERENCES}

AL-Ani, A. M. and Hancock, J. W. (1991) J. Mech. Phys. Solids 39, 23.

Betegón, C. and Hancock, J. W. (1991) J. Appl. Mech. 58, 104. 
Bilby, B. A., Cardew, G. E., Goldthorpe, M. R. and Howard, I. C. (1986) Size Effects in Fracture, p. 37. Institution of Mechanical Engineers, London.

ChaO, Y. J., YanG, S. and Sutton, M. A. (1991) Asymptotic Analysis of the Crack Tip Fields to Determine the Region of Dominance of the HRR Solutions. Presented at the 28th Annual Technical Meeting of the Society of Engineering Science, November 1991, Gainesville, Florida.

Du, Z.-Z. and Hancock, J. W. (1991) J. Mech. Phys. Solids 39, 555.

Harlin, G. and Willis, J. R. (1988) Proc. R. Soc. Lond. A 415, 197.

HutChinson, J. W. (1968) J. Mech. Phys. Solids 16, 13.

Hutchinson, J. W. (1983) J. Appl. Mech. 50, 1042.

Kirk, M. T., Koppfnhoefer, K. C. and Shin, C. F. (1991) Effect of Constraint on Specimen Dimensions Needed to Obtain Structurally Relevant Toughness Measures. ASTM-STP. In press.

Larsson, S. G. and Carlsson, A. J. (1973) J. Mech. Phys. Solids 21, 263.

Li, S. T. (1988) Master's Thesis, Institute of Mcchanics, Chinese Academy of Sciences (in Chinese).

LI, Y. C. and Wang, T. C. (1986) Scientia Sinica A29, 941.

LI, S. T. and WANG, T. C. (1992) Scientia Sinica A4, 395.

McClintock, F. A. (1971) Fracture: An Aduanced Treatise, Vol. III (ed. H. Liebowitz), p. 47. Academic Press, New York.

McMeEking, R. M. (1977) J. Mech. Phys. Solids 25, 357.

McMeeking, R. M. and PArks, D. M. (1979) Elastic-Plastic Fracture Mechanics, ASTMSTP-668, p. 175. American Society for Testing and Materials, Philadelphia, PA.

Needleman, A. and Tvergand, V. (1983) Elastic-Plastic Fracture: Second Symposium Volume I-Inelastic Crack Analysis, ASTM-STP-803, p. 80. American Society for Testing and Materials, Philadelphia, PA.

O'Dowd, N. P. and Shin, C. F. (1991) J. Mech. Phys. Solids 39, 989.

O'Dowd, N. P. and Shiн, C. F. (1992a) J. Mech. Phys. Solids 40, 939.

O'Dowd, N. P. and Shin, C. F. (1992b) Two-Parameter Fracture Mechanics: Theory and Applications. In press.

Parks, D. M. (1992) Topics in Fracture and Fatigue (ed. A. S. Argon), p. 59. Springer, New York.

Rice, J. R. (1968) J. Appl. Mech. 35, 379.

Rice, J. R. (1974) J. Mech. Phys. Solids 22, 17.

Rick, J. R. and Johnson, M. A. (1970) Inelastic Behavior of Solids (eds M. F. Kanninen et al.), p. 641. McGraw-Hill, New York

Rice, J. R. and Rosengren, G. F. (1968) J. Mech. Phys. Solids 16, 1.

Ritchie, R. O., Knott, J. F. and Rice, J. R. (1973) J. Mech. Phys. Solids 21, 395.

Ritchie. R. O. and Thompson, A. W. (1985) Met. Trans. A 16A, 233.

Sharma, S. M. and Aravas, N. (1991) J. Mech. Phys. Solids 39, 1043.

Shin, C. F. and German, M. D. (1981) Int. J. Fracture 17, 27.

SHin, C. F., O'Dowd, N. P. and Kirk, M. T. (1991) ^ Framework for Quantifying Crack Tip Constraint. ASTM-STP. In press.

Symington, M., Shih, C. F. and Ortiz, M. (1988) Tables of Plane Strain Mixed-mode Plastic Crack Tip Fields. Brown University Report. MRG/DMR-8714665/1, October.

Williams, M. L. (1957) J. Appl. Mech. 24, 111.

Xia, L. and Wang, T. C. (1992) Acta Mechanica Sinica 8, 156.

\section{APPENDIX}

Using (3.19), the plastic strains can be written as,

$$
\begin{gathered}
\tilde{\varepsilon}_{r_{3}}^{\mathrm{p}}=A\left(\tilde{\sigma}_{r_{7}}-\tilde{\sigma}_{\theta_{3}}\right)+B \tilde{\tau}_{, \theta_{3}}, \\
\tilde{\varepsilon}_{r_{3}}^{\mathrm{n}}=B\left(\tilde{\sigma}_{r_{3}}-\tilde{\sigma}_{\theta_{3}}\right) / 2+C \tilde{\tau}_{r \theta_{3}},
\end{gathered}
$$


where

$$
\begin{aligned}
& A=\Omega\left\{\left[(n-1) \tilde{S}^{2}+\tilde{T}\right] \tilde{T}^{2}\right\}, \\
& B=\Omega\left\{2(n-1) \tilde{\tau}_{r \theta_{1}} \tilde{S} \tilde{T}^{2}\right\}, \\
& C=\Omega\left\{2\left[(n-1) \tilde{\tau}_{r \theta_{1}}^{2}+\tilde{T}\right] \tilde{T}^{2}\right\}, \\
& \tilde{S}=\frac{1}{2}\left(\tilde{\sigma}_{r_{1}}-\tilde{\sigma}_{i_{1}}\right), \\
& \tilde{T}=\tilde{S}^{2}+\tilde{\tau}_{r \theta_{1}}^{2}, \\
& \Omega=\frac{3^{(n+1) ; 2}}{4} \tilde{T}^{(n-7) / 2} .
\end{aligned}
$$

The coefficients $D_{1}-D_{5}$ in (4.7) are given below:

$$
\begin{aligned}
D_{1}= & A, \\
D_{2}= & 2 A^{\prime}-\left(n s_{1}+\Delta s_{3}+s_{3}+2\right) B, \\
D_{3}= & A^{\prime \prime}-\left(n s_{1}+\Delta s_{3}+2 s_{3}+3\right) B^{\prime}-\left[\left(n s_{1}+\Delta s_{3}\right)\left(n s_{1}+\Delta s_{3}+2\right)\right. \\
& \left.+\left(s_{3}+2\right) s_{3}\right] A+2\left(n s_{1}+\Delta s_{3}+1\right)\left(s_{3}+1\right) C, \\
D_{4}= & -\left(s_{3}+1\right) B^{\prime \prime}-2\left(s_{3}+2\right) s_{3} A^{\prime}+\left[\left(n s_{1}+\Delta s_{3}\right)\left(n s_{1}+\Delta s_{3}+2\right)\left(s_{3}+1\right)\right. \\
& \left.+\left(n s_{1}+\Delta s_{3}+1\right)\left(s_{3}+2\right) s_{3}\right] B+2\left(n s_{1}+\wedge s_{3}+1\right)\left(s_{3}+1\right) C^{\prime}, \\
D_{5}= & -\left(s_{3}+2\right) s_{3} A^{\prime \prime}+\left(n s_{1}+\Delta s_{3}\right)\left(n s_{1}+\Delta s_{3}+2\right)\left(s_{3}+2\right) s_{3} A \\
& +\left(n s_{1}+\Delta s_{3}+1\right)\left(s_{3}+2\right) s_{3} B^{\prime} .
\end{aligned}
$$

